\title{
Endoneurosurgical hemostasis techniques: lessons learned from 400 cases
}

\author{
Amin Kassam, M.D., Carl H. Snyderman, M.D., Ricardo L. Carrau, M.D., \\ Paul Gardner, M.D., AND Arlan Mintz, M.SC., M.D. \\ Minimally Invasive Endoneurosurgical Center, University of Pittsburgh Medical Center, Pittsburgh, \\ Pennsylvania
}

\begin{abstract}
The increasing popularity of minimally invasive neurosurgery has led to the development of transnasal expanded approaches for the treatment of skull base lesions. One of the greatest challenges in safely accomplishing resection of tumors, particularly intradural lesions, is effective hemostasis. Over the past 7 years the authors have progressively developed an organized approach to address this challenge. This has required the development of new instrumentation as well as variations on standard techniques. In this report they present the technique that has evolved at their institution for endoneurosurgical hemostasis.
\end{abstract}

\author{
KEY WORDS • hemostasis • hemorrhage • arterial bleeding • venous bleeding • \\ endoscopy • transsphenoidal approach
}

For the purpose of this report we will define endoneurosurgery as intradural surgery performed using parallel rodlens endoscopy as the sole visualization tool, in contradistinction to through-channel endoscopy (ventriculoscopy) or microsurgery. Endoneurosurgery can be classified into expanded endonasal approaches or endoport surgery depending on the corridor used; that is, paranasal sinus or transcortical approaches, respectively., ${ }^{1,2}$ We will discuss the techniques used to achieve hemostasis in each of these endoneurosurgical approaches together because they are identical, relying on the concept of corridor surgery. With the increasing popularity of expanded endonasal approaches over the past decade, the ability to achieve intraoperative hemostasis is one of the greatest concerns for surgeons contemplating endoneurosurgical procedures. It is essential that surgeons specializing in endoscopy become comfortable with a variety of hemostatic techniques before performing intradural procedures.

Prevention of bleeding is obviously the best solution. Dissection techniques that minimize the risk of vessel injury during tumor removal should be adopted. The principles of endoneurosurgical dissection are identical to those of microsurgical dissection; specifically, tumor debulking, extracapsular sharp dissection, and countertraction using gentle suction. The absolute avoidance of pulling takes precedence over all other considerations, including the extent of tumor removal. Nevertheless, vascular injuries may occur not only because of excessive pulling on tumor tissues but also from inadequate visualization of dissection planes, blind instrumentation, direct or indirect injury (thermal injury) from powered instruments (drills, microdebriders, ultrasonic aspirators), and tumor vascularity. Despite application of an ideal technique, bleeding is often unavoidable and hemostasis is required to deal with both planned and unplanned events.

We have found it useful to categorize bleeding based on two factors; the source (venous or arterial) and the rate (high-flow or low-flow bleeding). Venous bleeding can involve low flow, as is the case of diffuse generalized oozing from the mucosa, or it can be high-flow focal bleeding from the cavernous sinus. Similarly, arterial bleeding can be low flow, as occurs from small perforating vessels, or high flow, as is the case with carotid artery injuries.

The choice of hemostatic techniques not only depends on the source and rate of the hemorrhage but is also influenced by factors such as the type of tissue (mucosa, bone, and tumor); the proximity of neurovascular structures; and the area (extradural or intradural) of dissection. At the outset it is important to mention that the principles of endoneurosurgical hemostasis are identical to those of microsurgical hemostasis, and the reader may find these to be obvious and repetitive. We do not wish to redevelop these time-tested principles, however, but to rather to demonstrate that they are completely applicable and, with some modifica- 
tions, are effective in establishing endoneurosurgical hemostasis. In this paper we will present an algorithm for endoneurosurgical hemostasis based on the factors discussed earlier and our learning curve over the past 7 years.

\section{ENDONEUROSURGICAL HEMOSTASIS}

\section{Equipment and Hemostatic Materials}

Before discussing hemostatic techniques it is important to be aware of the equipment and materials available. The most important equipment for the endoneurosurgeon is the bipolar electrocautery. Standard bayonet designs are too large for a transnasal route, especially for expanded endonasal approaches; instead, a pistol-grip design should be used. There are five endobipolar cautery devices available, which vary based on the fineness and configuration of the distal tip (Fig. 1). Therefore, the appropriate bipolar device can be selected based on the geometry of the vessel, its site, caliber, and proximity to critical neurovascular structures. This allows selective cauterization of tissues with minimal thermal spread to surrounding areas. Monopolar cautery can be used only on intranasal tissues and should not be used within the sphenoidal sinus, on the skull base, or intracranially. The risk of thermal dispersion in these confined areas, and of creating neurovascular injuries, is significant. Special hemoclip appliers are available that are designed for endoscopic use.

A variety of hemostatic materials are available. All of these products require direct contact with the bleeding tissues and work by promoting platelet aggregation and formation of a stable clot. Examples of such hemostatic materials include Avitene (Ethicon [Johnson and Johnson], Somerville, NJ), Floseal (Baxter International, Inc., Deerfield, IL), and Syvek (Marine Polymer, Boston, MA). These materials are most effective for venous hemostasis and low-flow arterial bleeding. An important consideration in selecting and delivering the specific agent is the need to maintain visualization within these corridors. We have found that the application of dry Avitene to the bleeding site with gentle pressure is the most effective means of achieving hemostasis without interfering with visualization of the rest of the surgical field.

Special mention should be made of warm water irrigation. It is a common misconception that cold water irrigation provides the best hemostasis. Nevertheless, the amount of vascular constriction that occurs with cold water is insufficient to provide effective hemostasis. Clinical trials of hot water irrigation, in contrast, show that it is nearly as effective as surgical techniques for the treatment of epistaxis. ${ }^{3}$ The ideal temperature for coagulation has been determined to be approximately $40^{\circ} \mathrm{C} .{ }^{4}$ We have found, however, that irrigation with saline that is just warm to the touch is adequate. A number of different mechanisms for this method of hemostasis have been proposed, including activation of platelet aggregation, enhanced coagulation, and interstitial edema. Warm saline irrigation is performed whenever there is diffuse oozing, either from traumatized nasal mucosa, denuded bone surface, or the dissected surface of the tumor or brain. Gentle irrigation of the brain surface with a soft catheter is effective in clearing the field so that bleeding sites can be visualized and selectively cauterized or compressed with a hemostatic material. Care should be taken to
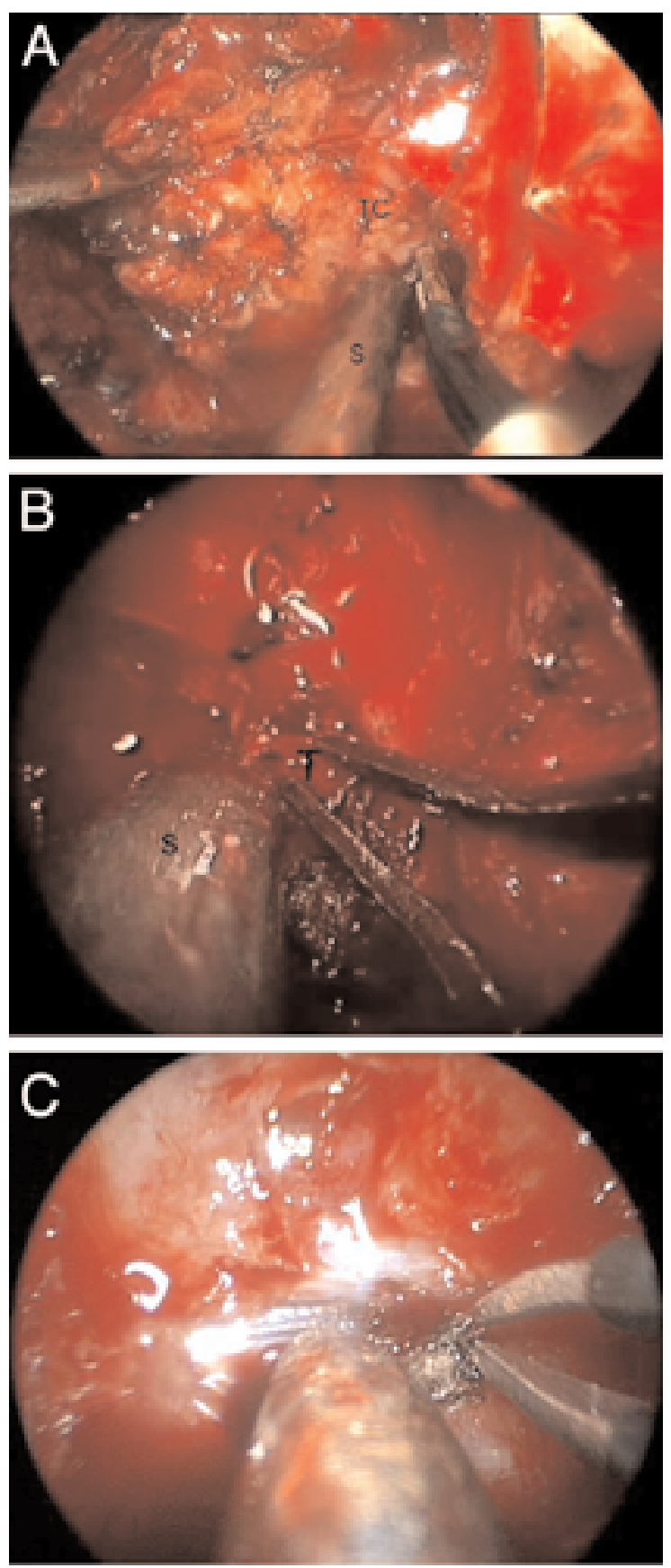

Fig. 1. Intraoperative views demonstrating the different types of endonasal bipolar cautery devices. A: Endoscopic view obtained during endonasal resection of an olfactory groove meningioma. The bipolar devices used have the two blades placed along a vertical plane with insulation close to the distal end. These devices are most useful for coagulating the tumor capsule (TC) during the dissection. The suction ( $\mathrm{S}$ ) is brought close to the target to provide good visualization. B: Endonasal view obtained during endoscopic resection of a clival chordoma, demonstrating a more aggressive bipolar cautery with the blades oriented along a horizontal plane and bent approximately $30^{\circ}$ to improve visualization. The devices are not fully insulated up to the distal tip, and in sealing the tear $(\mathrm{T})$ in the vessel the tissue between the noninsulated portion of the proximal tips is also coagulated. C: Endoscopic view demonstrating the fine endoscopic bipolars. Note the insulation extends to allow for focal exposure of the fine tips preventing heat dispersion. 
avoid excessive irrigation pressures because hydrodissection of the tissues could occur.

Specific hemostasis techniques have been developed for both venous and arterial bleeding that is encountered during expanded endonasal approaches. These techniques are as follows.

\section{VENOUS HEMOSTASIS}

\section{Low-Flow Bleeding}

Diffuse venous bleeding from mucosa and bone responds very well to warm saline irrigation, and this is our preferred method. For extradural application, this is delivered with a bulb syringe; for intradural application, an external ventricular drain catheter or similar soft catheter is used. Focal bleeding from bone may be controlled by drilling with a diamond burr or application of bone wax with a pledget.

Focal venous bleeding from the cavernous or another venous sinus can be quite brisk and the rapidity of blood loss can be underestimated because of the clearance of blood with the suction device. Constant communication with the anesthesiologist is essential so that volume loss can be anticipated and blood replacement can be instituted. It is sometimes necessary to work through the blood loss until tumor dissection in the cavernous sinus is completed. We believe that focal bleeding from the cavernous sinus is best controlled with precision application of Avitene on a pledget. The pledget must be dry, with the Avitene placed on the inside, creating a "sandwich" of the substance (Fig. 2). This is applied directly to the bleeding point and may need to be replaced with exchanges multiple times until bleeding is controlled.

The technique for the exchange requires two surgeons, because Avitene can only be effective if it is directly and precisely applied over the focal site of the bleeding. The surgeon performing the dissection brings the "sandwich" into the field and prepares it for positioning. Once it is close to the target, the surgeon handling the endoscope then removes the previous "sandwich," allowing the surgeon performing the dissection to use a suction device with one hand while laying the replacement "sandwich" on the target (Fig. 2, Video 1). It is best to treat each bleeding source as it arises rather than trying to deal with multiple bleeding sites at the same time. Venous bleeding from large veins is halted using the technique for low-flow arterial bleeding described later.

Video 1. Technique for Avitene "sandwich" exchange. (Clich here to view with Windows Media Player and a broadband connection here to view with a dial-up connection, or here to view with RealPlayer.)

\section{ARTERIAL HEMOSTASIS}

\section{High-Flow Arterial Bleeding}

In the case of high-flow bleeding it is critical to confine the flow to a specific portion of the artery to allow for the direct application of bipolar coagulation. Blood has a tremendous capacity for heat dispersion; therefore, diffuse cauterization without identifying the small segment of the vessel with the laceration will not be effective. The suction device is used to isolate the bleeding site by maintaining a dry field and directing the stream of blood away from the tip of the endoscope. Once the hole in the artery can be seen clearly, the bipolar electrocautery is applied along the sidewall of the vessel (Fig. 3). The specific type of endoneurosurgical bipolar device chosen will vary depending on the factors previously discussed. Gentle irrigation with saline helps prevent sticking of the bipolar electrocautery blades to the vessel.

A critical difference in comparison with microsurgery is the ability to maintain visualization by using the endoscope during the course of this type of bleeding. This requires a significant amount of collective experience among the team members to be able to navigate through the bleeding and maintain vision. Although the principles are the same, with the surgeon who is performing the dissection managing the two primary suction devices while the endoscopist uses a third one to follow the flow of blood to the focal site, a key difference is the need for two surgeons to maintain visualization (Video 2).

Video 2. Technique for bipolar cautery in high-flow arterial bleeding. (Click here to view with Windows Media Player and a broadband connection, here to view with a dial-up connection, on here to view with RealPlayer.)

In cases involving these larger vessels with high-flow hemorrhage, the immediate and delayed consequences of
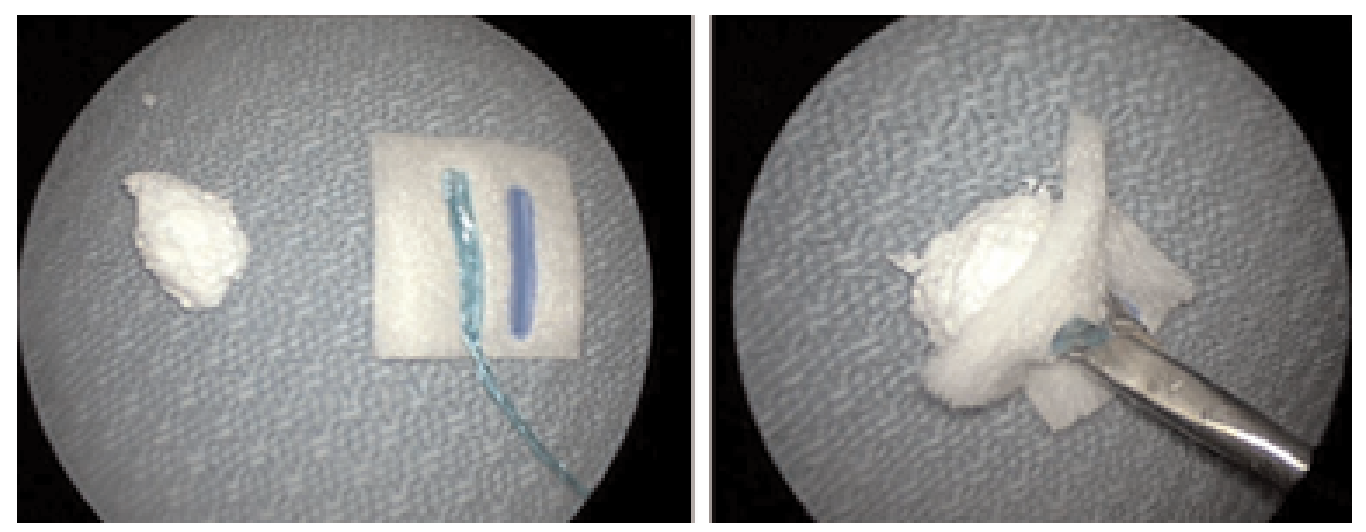

Fig. 2. Photographs showing preparation of the Avitene "sandwich." 
the thermal injury to the vessel wall that occurs during the coagulation needs to be borne in mind. The probability of immediate occlusion of the vessel is extremely high and the tolerance of the distal circulation will be contingent on the collateral supply. Therefore, an assessment of this eventuality must be considered during perioperative and intraoperative planning. The surgeon must be very vigilant for the delayed sequelae of thermal injury and the development of a pseudoaneurysm. Therefore, early and delayed postoperative arteriograms are important.

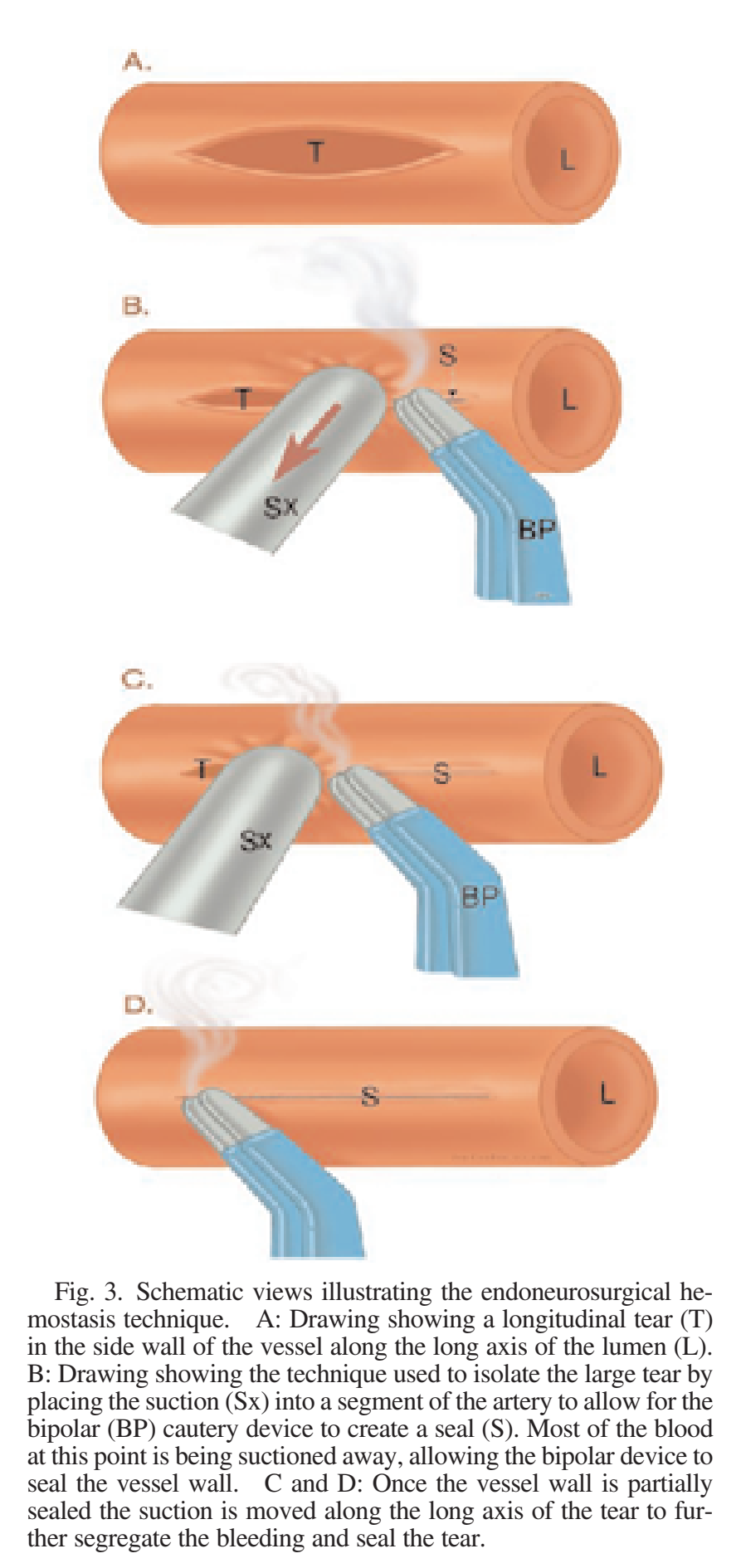

\section{Low-Flow Arterial Bleeding}

For low-flow bleeding from small arterioles, the technique is similar, with selective use of bipolar electrocautery. One should bear in mind, however, that small vessels such as perforating arteries from which this type of bleeding is often sustained are in critical locations, and so heat dispersion to adjacent structures becomes a paramount issue. Continued irrigation with saline during cauterization helps disperse the heat. In these low-flow hemorrhages, isolated segmental packing can be judiciously used. There are a variety of hemostatic materials such as Avitene and Syvek (off-label indication) that we have found useful. Nevertheless, extreme caution must be exercised here. We do not favor this approach unless the packing can be applied focally with no possibility that the vessel will retract or allow bleeding to continue in a retrograde fashion into the adjacent brain. This will inevitably lead to parenchymal hemorrhage, with devastating consequences. Therefore, we rarely use this technique and do so only when these contraindications are not present. Furthermore, if packing is required it is positioned just on the affected vessel to allow visualization of all of the surrounding neural tissue. Only enough pressure is maintained to tamponade the bleeding while still being able to see the normal physiological pulsations in the surrounding brain (Video 3).

Video 3. Technique for halting low-flow bleeding from arterioles $\left(\mathrm{P}_{1}\right.$ perforating artery). (Click here to view with Windows Media Player and a broadband connection, here to view with a dial-up connection, on here to view with RealPlayer.)

\section{ADDITIONAL SPECIAL CONSIDERATIONS}

\section{Carotid Artery Injury}

When a major carotid artery injury occurs, the immediate concern is maintaining visualization so that the actual defect can be seen. This requires the active participation of both surgeons with the use of two suction devices in addition to the endoscope and dissection instrument. Pressure is applied with a pledget to achieve immediate control. The anesthesiologist takes appropriate measures to maintain blood pressure and restore intravascular volume. A hypotensive state should be avoided; the blood pressure should be high enough to maintain collateral cerebral perfusion. Neurophysiological monitoring may provide early evidence of inadequate perfusion.

In our opinion, every effort should be made to control the bleeding intraoperatively. Indiscriminate packing should be avoided because this will not prevent bleeding intracranially. The edge of the bleeding site is then cauterized with bipolar electrocautery as the pledget is moved aside. Only if the bleeding cannot be stopped with these measures is the site packed focally and the patient transported to the angiography suite for placement of a stent if possible or permanent occlusion of the vessel if not. If adequate collateral circulation is not present, a vascular bypass procedure is performed in the same session.

\section{Devascularization of Tumor Tissues}

Preoperative strategies include devascularization of the 
tumor by angiographically guided embolization of the vascular bed when possible. The majority of tumors in this location receive their blood supply from small feeding vessels that can be difficult to cannulate for direct embolization with particulate matter. In the absence of direct cannulation, the use of alcohol embolization has been suggested. Based on our experience, we have abandoned the use of intraarterial infiltration of the tumor bed with alcohol. Although it is effective, we have observed a number of complications related to diffusion of the alcohol to critical vascular territories; these include secondary strokes of the cortex and ischemic injury to the contents of the cavernous sinus.

With the use of the expanded endonasal approach, we have been able to gain circumferential access to these lesions, obviating the need for this type of embolization in the majority of cases, even in tumors that are known for being hypervascular (angiofibromas, meningiomas, paragangliomas). With large intranasal lesions, debulking of the tumor to the level of the skull base may be necessary to obtain access to the remaining blood supply. Devascularization of tumors can be achieved by ligation of feeding arteries. For tumors in the sinonasal space, the key arterial supply is from the internal maxillary artery and its branches. The critical branches are the sphenopalatine and posterior nasal arteries, which can be accessed early. These vessels exit the sphenopalatine foramen at the posterior-superior corner of the maxillary sinus, deep to the attachment of the middle turbinate. A maxillary antrostomy is made and a subperiosteal dissection is performed to identify the foramen. Overlying bone is removed with a 1-mm angled Kerrison rongeur to expose the main trunk of the vessel before the branching point, and a hemoclip is placed.

Tumors that involve the anterior cranial base often receive the majority of their blood supply from the anterior and posterior ethmoid arteries. A complete ethmoidectomy is first necessary to expose the roof of the ethmoid sinus. The anterior ethmoid artery crosses the skull base just posterior to the nasofrontal recess. The thin lamina papyracea of the medial orbital wall is fractured and removed up to the plane of the cranial base. Further elevation of the periosteum on the orbital side exposes the vessel where it leaves the orbit, and a hemoclip can be placed (Fig. 4). The posterior ethmoid artery is similarly ligated in the roof of the posterior ethmoid air cells.

The expanded endonasal approach provides early exposure of the dural base, facilitating further devascularization in the case of anterior base lesions. The best example of this is anterior base meningiomas, which receive a blood supply from the dural base in addition to the ethmoid arteries. It is not uncommon in anterior skull base meningiomas to find an additional feeding artery arising from the distal portion of this paraclinoid carotid at the level of the opticocarotid recess. This vessel should be specifically identified and coagulated. Care should be taken to prevent the proximal stump from bleeding and retracting into the orbit, resulting in a retrobulbar hematoma.

\section{DISCUSSION}

The principles discussed earlier are identical to those applied in achieving hemostasis when using an operating microscope. In this paper we have provided some important
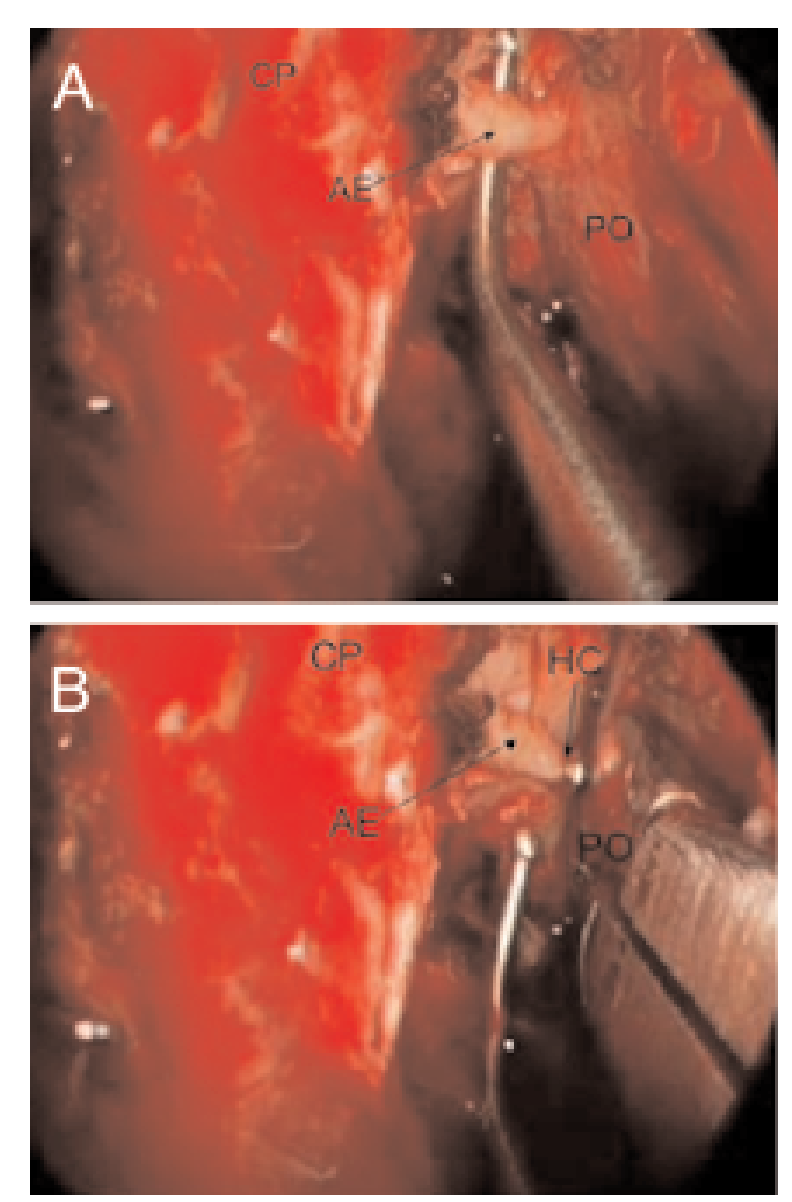

Fig. 4. A: Endoscopic endonasal view demonstrating the isolation of the left anterior ethmoidal artery (AE). The vessel can be seen exiting the periorbita (PO) laterally and running into the cribriform plate (CP) medially. The lamina papyracea has been removed, exposing the periorbita, and wide bilateral ethmoidectomies have been undertaken. B: Endoscopic view demonstrating the position of the hemoclip (HC) directly at the point of exit of the anterior ethmoidal artery from the periorbita.

considerations that are important in executing these principles when using an endoscope to achieve hemostasis. The most common concerns raised when discussing the ability to effect endoneurosurgical hemostasis have centered on the lack of true three-dimensional visualization and the necessity of working in a relatively narrow corridor at a distance. Together these concerns have raised questions about the ability to maintain visualization, particularly during high-flow arterial bleeding.

To address these questions, several key endoneurosurgical principles and critical equipment used in the procedure have been emphasized throughout this paper. The most important of these is the concept of team surgery, which represents perhaps the most fundamental difference from microsurgery. It is our opinion that in situations in which bleeding is likely to be encountered, team surgery is mandatory. The value of having a team of surgeons working simultaneously is most evident when a vascular crisis occurs. A single surgeon cannot hold an endoscope, suction blood, and achieve hemostasis without help. A stationary 
endoscope holder may suffice during uncomplicated dissection but is woefully inadequate if an artery is squirting toward the end of the endoscope. With profuse hemorrhage, it may even be necessary to use two suction devices simultaneously to maintain visualization. An inexperienced assistant is unlikely to be helpful in such a situation.

Currently the most significant limitation of endoneurosurgical hemostasis is the inability to repair large arteries primarily. Endoscopic suturing techniques are not yet available to reconstruct tears in large vessels primarily. This represents a major difference when compared with microsurgery. Endoneurosurgical techniques for application of vascular clips, including encircling clips, are currently available. Nevertheless, we will need to develop techniques and instruments to reconstruct large vessels in those rare situations in which this is warranted.

\section{CONCLUSIONS}

Endoscopic hemostatic techniques must be mastered to perform effective and safe endoneurosurgery. The risk of vascular complications is minimized with adequate preoperative preparation, sound anatomical knowledge, proper dissection techniques, familiarity with hemostatic materials, and treatment algorithms for emergency situations.
Based on our experience with 400 cases between 1998 and 2005, we have found the algorithm described to be an effective means of securing hemostasis during endoscopic endonasal surgery for both intra- and extradural lesions.

\section{References}

1. Harris AE, Hadjipanayis CG, et al: Microsurgical removal of intraventricular lesions using endoscopic visualization and stereotactic guidance. Neurosurgery 56 (Suppl 1):S-125-S-132, 2005

2. Kassam AB, Snyderman C, Gardner P, et al: The expanded endonasal approach: a fully endoscopic transnasal approach and resection of the odontoid process: technical case report. Neurosurgery 57 (Suppl 1):E213-E214, 2005

3. Stangerup SE, Dommerby H, Lau T: Hot-water irrigation as a treatment of posterior epistaxis. Rhinology 34:18-20, 1996

4. Stangerup SE, Thomsen HK: Histological changes in the nasal mucosa after hot-water irrigation. An animal experimental study. Rhinology 34:14-17, 1996

Manuscript received June 8, 2005.

Accepted in final form June 28, 2005.

Address reprint requests to: Amin B. Kassam, M.D., University of Pittsburgh School of Medicine, 203 Lothrop Street, Suite 500, Pittsburgh, Pennsylvania 15213. email: kassamab@upmc.edu. 